Apidologie, 1982, 13 (1), 29-37.

\title{
CHROMATOGRAPHIE EN PHASE GAZEUSE DES COMPOSÉS VOLATILS \\ DES GLANDES A PHÉROMONES DES ABEILLES : MÉTHODES D'ANALYSE DIRECTE
}

\author{
B. MAUCHAMP \\ Laboratoire Phytopharmacie, I.N.R.A., 78000 Versailles \\ D. GRANDPERRIN \\ Université P.-et-M.-Curie, E.R.A. 620 "Cytophysiologie \\ des Arthropodes ", 105, bd Raspail, 75006 Paris
}

\section{RÉSUMÉ}

Les phéromones des insectes sont des substances très volatiles. Les mauvais rendements des méthodes d'extraction et de purification nécessaires lors de leur étude en chromotographie imposent un grand nombre de dissections. Cet article présente un équipement très simple permettant l'introduction directe et en petit nombre des glances à phéromones à l'entrée d'une colonne à chromatographie; le procédé a permis l'analyse de la substance d'alarme du dard de l'ouvrière d'Abeille (Apis mellifica L.).

Mots clés : chromatographie phase gazeuse, glande, phéromone, substance d'alarme, abeille ouvrière.

\section{INTRODUCTION}

La vie des sociétés d'Insectes est contrôlée, hormis les facteurs externes (climat, photopériode, nourriture) et génétiques, par des phéromones. Ces substances sont des composés volatils de faible poids moléculaire élaborées par des glandes de dimensions réduites. Elles sont produites par les individus de toutes les castes et émises en très faible quantité $\left(10^{-6}\right.$ à $10^{-12} \mathrm{~g}$ par individu); elles provoquent chez les congénères une réaction spécifique au plan comportemental (attraction, répulsion...) et (ou) physiologique (substance royale des reines d'abeilles).

Le caractère très volatil de ces composés et leur faible concentration ont imposé l'adaptation et l'utilisation des méthodes d'analyse les plus fines. Manipuler à l'aide 
d'un solvant rend nécessaires un abondant matériel biologique et un grand nombre de dissections (BUTENANDT et al., 1959). Cependant, il est possible d'utiliser un nombre limité d'Insectes en introduisant directement une ou quelques glandes au niveau de l'injecteur d'un appareil de chromatographie en phase gazeuse (StÄLlBERG-STENHAGEN, 1972; Bergström, 1973; Morgan et al., 1979; Descoins et Gallois, 1979). Les équipements précédemment décrits sont néanmoins difficilement utilisables sur tous les modèles de chromatographes. Aussi, nous avons adapté et réalisé un dispositif simple, peu onéreux, valable sur tout appareil.

\section{MATÉRIEL ET MÉTHODES}

Les analyses sont réalisées par chromatographie en phase gazeuse à l'aide d'un appareil Girdel série 30 muni d'un détecteur à ionisation de flamme. La séparation est obtenue grâce à une colonne pleine ( $5 \%$ F.F.A.P. sur Q.C.Q. 100-120) de $2,5 \mathrm{~m}$. La température du four est programmée de $50^{\circ} \mathrm{C}$ à $160^{\circ} \mathrm{C}$ (élévation de $\left.10^{\circ} \mathrm{C} / \mathrm{mn}\right)$.

\section{Dispositif d'introduction de l'échantillon}

Ce dispositif a été conçu pour introduire les échantillons à analyser au niveau de l'injecteur sans perturber le débit du gaz vecteur. A tout instant, ce système permet une isolation totale avec l'extérieur; il est constitué de trois parties principales (fig. 1).

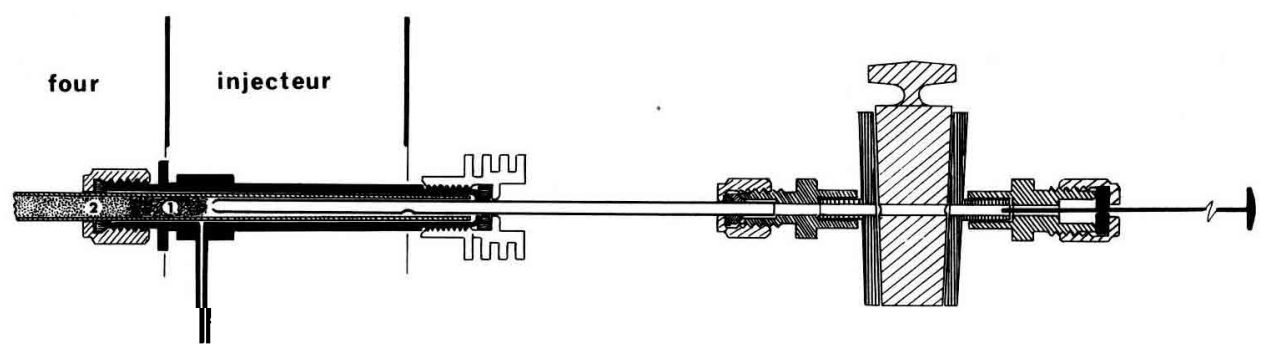

FIG. 1. - Vue d'ensemble du dispositif une fois en place dans le chromatographe en phase gazeuse. Un tampon de laine de verre (1) évite la dispersion des bris de verre après rupture de la capsule et protège la phase (2) de la colonne.

Fig. 1. - General view of the device in position in the gas chromatograph. A plug of glass-woll (1) avoids the dispersion of pieces after the breaking of the seal and protects the phase (2) of the column.

- L'aiguille d'introduction: C'est un tube en acier inoxydable de $13 \mathrm{~cm}$ de long et $3 \mathrm{~mm}$ de diamètre interne directement introduit en tête de colonne. L'extrémité de l'aiguille est ouverte mais présente une constriction bloquant la capsule contenant les glandes. Des ouvertures latérales pratiquées sur la partie logée dans la colonne (fig. 2) assurent à l'intérieur de l'aiguille un bon balayage du gaz vecteur.

Ce tube est introduit dans l'injecteur au travers du septum d'injection directe présent sur la plupart des chromatographes. Pour cela, la perforation du septum est légèrement agrandie $(2 \mathrm{~mm})$. 


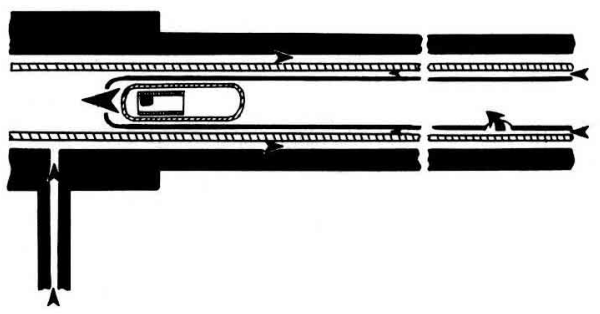

FIG. 2. - Vue de détail de l'extrémité de l'aiguille d'injection et de la capsule. Les flèches indiquent le trajet du gaz vecteur.

FIG. 2. - Detailed view of the point of the injection needle and of the seal. Arrows show the path of the vector gas.

La simplicité de ce système de raccordement avec l'injecteur facilite le démontage et autorise une utilisation de l'appareil pour des injections classiques.

- Le boisseau : Il a rôle de sas d'introduction.

Deux raccords sont soudés en ligne. L'un assure la jonction entre le corps du boisseau et l'aiguille d'in-. troduction, l'une porte le nouveau spetum au travers duquel se déplace le piston poussant la capsule dans l'aiguille d'introduction.

Une troisième ouverture est aménagée sur le corps du boisseau. Ces trois orifices, ainsi qu'un évidement traversant l'axe mobile du boisseau sont situés dans le même plan.

- Le piston : Il permet de pousser la capsule jusqu'à l'extrémité de l'aiguille et de l'y casser.

L'ensemble est maintenu par un étrier. Son montage s'effectue en quelques minutes. Il est d'un entretien facile et toute contamination peut être évitée ou éliminée en nettoyant les différentes parties à l'aide d'un solvant.

- L'ampoule d'introduction: L'ampoule est un tube de verre ètiré de $1,5 \mathrm{~mm}$ de diamètre scellé à ses deux extrémités. Sa longueur doit être compatible avec les mouvements de l'axe mobile du boisseau. Elle contient les glandes et doit être fermée rapidement à la flamme d'un microchalumeau. Pour éviter les risques de pyrolyse ou d'évaporation lors de la fermeture, les glandes disséquées peuvent être regroupées dans un microcapillaire court ( $2 \mathrm{~mm}$ de long), lui-même introduit dans la capsule.

Ce capillaire joue le rôle de volant thermique et évite le contact direct du matériel biologique avec la paroi de l'ampoule (site d'une élévation de température).

Dans ces conditions, les solutions standard les plus volatiles sont conservées. Les ampoules scellées sont maintenues au congélateur jusqu'au moment de leur utilisation; si ces dernières sont conservées au froid, il est nécessaire de les ramener à température ambiante avant utilisation.

\section{Mode opératoire}

L'introduction d'une capsule s'effectue en deux temps. En premier lieu (fig. 3 a), l'ampoule est logée dans l'évidement de l'axe du boisseau (1) qui subit une rotation de $90^{\circ}$ (2) mettant la capsule dans l'axe de l'aiguille. A l'aide du piston (fig. $3 \mathrm{~b}$ ), l'ampoule est poussée progressivement (3) jusqu'à l'extrémité de l'aiguille où elle est laissée 1 à $2 \mathrm{mn}$ avant d'être cassée par le piston (temps zéro de l'injection) qui est ensuite retiré au-delà du boisseau dont l'axe mobile est remis en position (1).

\section{Résultats}

Le dispositif décrit a été utilisé et testé lors de l'analyse de la substance d'alarme émise au niveau du dard chez l'ouvrière d'abeille (Apis mellifica L.). BLuM et al. (1978), à partir d'extraits de dards entiers a identifié quelques composés nous permettant de définir les paramètres de l'analyse chromatographique et 


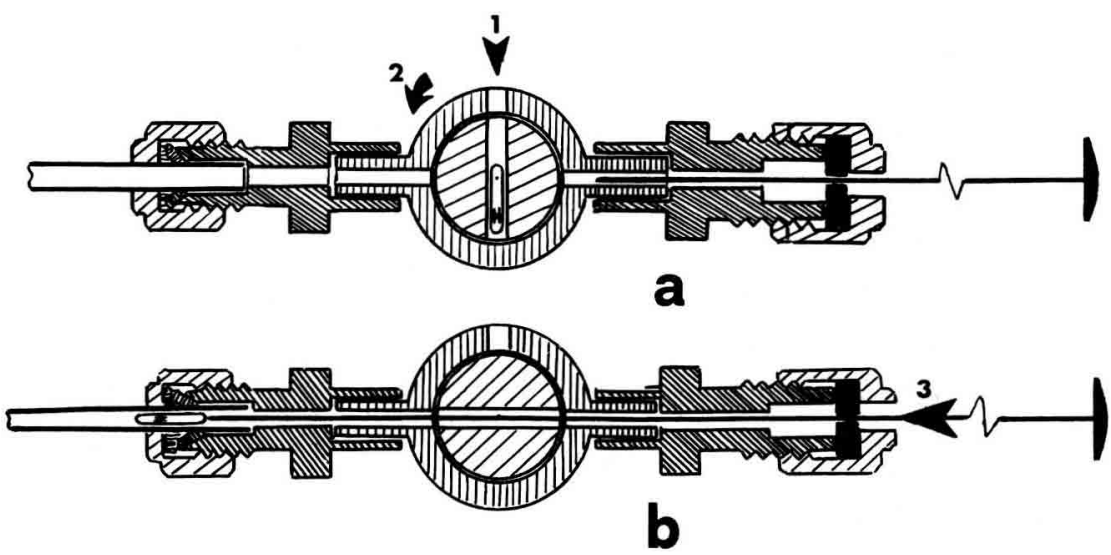

FIG. 3. - Détail de fonctionnement de l'injecteur. $a:$ mise en place de la capsule au niveau du boisseau. $b$ : refoulement de la capsule jusqu'à l'extrémité de l'aiguille.

FIG. 3. - Detail of the working of the injection. $a:$ setting of the seal at the faucet-pipe.

$b:$ pressing back of the seal to needle point.

de constater la parfaite reproductibilité des résultats obtenus lors des différentes injections d'un mélange des produits standards.

Le dard comporte plusieurs formations glandulaires susceptibles de participer à l'élaboration et à la sécrétion de ces composés.

Cette substance d'alarme est libérée au niveau d'une surface de stockage : la membrane poilue (MASCHWITZ, 1964) mais serait en réalité produite par les glandes de Koschewnikow (plate-gland) (GHENT et GARY, 1962) et de là s'écoulerait jusqu'à la membrane poilue (voir figure 4 et photographies 1 et 2) Nous avons donc effectué, dans un premier temps, les essais à partir de ces deux types de formations.

Les glandes et membranes poilues sont prélevées sur des ouvrières d'abeilles de race italienne (Apis mellifica ligustica) d'âge indéterminé tuées par le froid et disséquées sous binoculaire. Les pièces sont scellées en capsules.

Les résultats sont présentés sur la figure 5 .

Le chromatogramme donnant les pics de référence (fig. $5 a$ ) a été obtenu par injection d'un solvant (hexane) contenant en solution les composés identifiés par Blum et al. (1978). Parmi les produits volatils détectés au niveau de la membrane poilue (fig. $5 b$ ) et les glandes de Koschewnikow (fig. $5 c$ ), plusieurs ont pu être identifiés; ils correspondent à ceux déjà mentionnés (BLUM et al., 1978) et sont présents dans les deux formations mais à des concentrations très différentes puisque la figure $5 b$ correspond à une membrane poilue et la figure $5 c$ à 20 glandes de Koschewnikow.

Toutefois, les composés correspondants à certains pics n'ont pu être identifiés de façon absolue.

\section{DISCUSSION ET CONCLUSION}

Le dispositif décrit est simple, il peut s'adapter sur un grand nombre de chromatographes en phase gazeuse. Utilisé ici sur un appareil muni d'une colonne pleine, le modèle proposé peut être monté, en modifiant l'interface au niveau de l'injecteur, sur un 


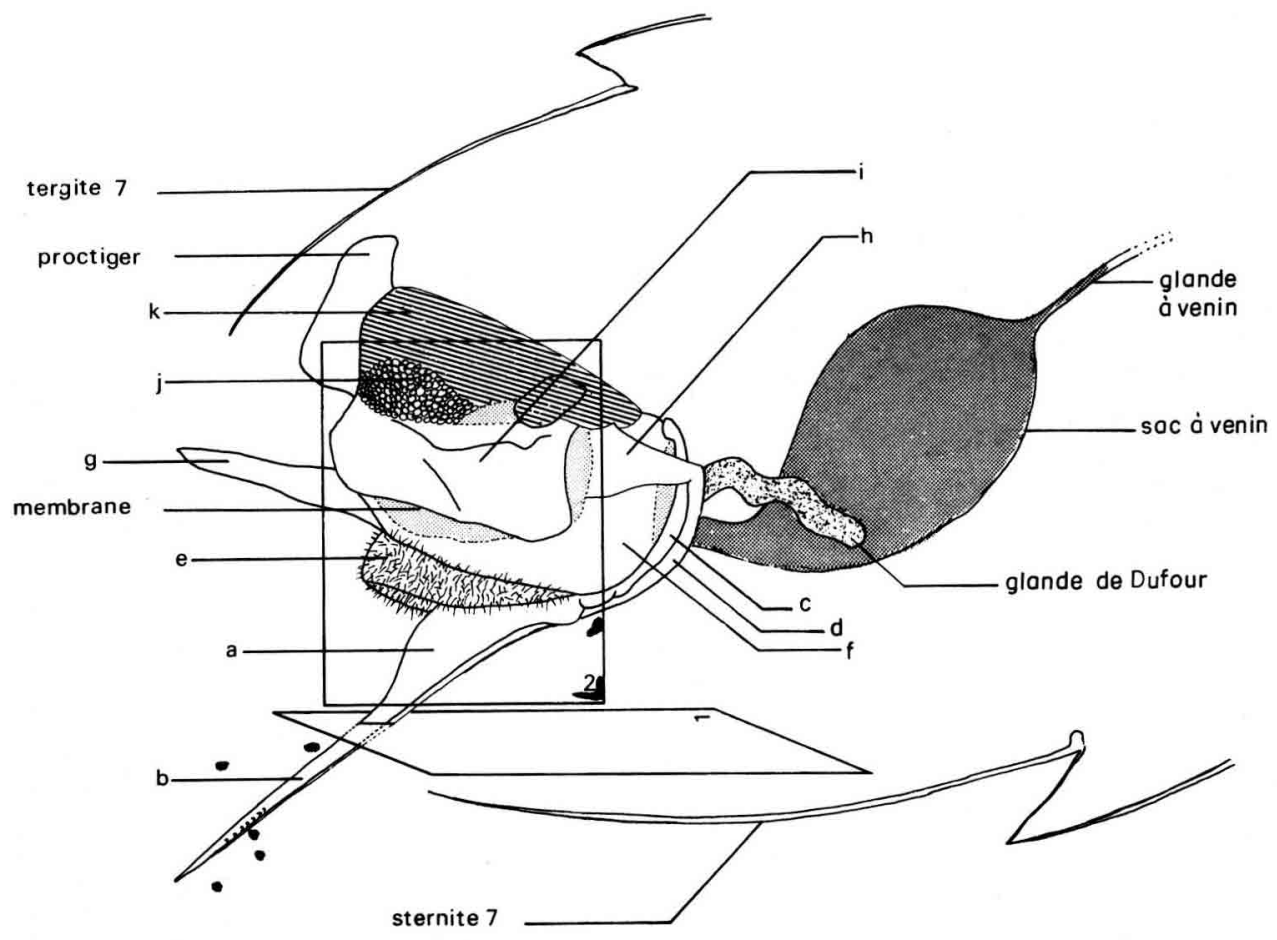

FIG. 4. - Vue latérale droite d'un dard d'ouvrière d'Abeille (Apis mellifica $L$.).

$a:$ bulbe de l'aiguillon; $b:$ aiguillon; $c:$ ramus $1 ; d:$ ramus $2 ; e:$ membrane poilue; $f:$ plaque oblongue; $g:$ gaine de l'aiguillon (anormalement repliée); $h:$ plaque triangulaire; $i:$ plaque quadratique;

$j$ : glande de Koschewnikow; $k$ : muscle.

FIG. 4. - Lateral right view of the honeybee worker sting (Apis mellifica $L$.).

$a:$ bulb of the stylet of the sting; $b:$ sting; $c:$ st ramus; $d: 2$ nd ramus;

$e:$ hairy membrane; $f:$ oblong plate; $g:$ sheath of sting (anormally folded up);

$h$ : triangular plate; $i$ : quadrate plate; $j:$ Koschewnikow gland; $k:$ muscle.

chromatographe comportant une colonne capillaire comme cela a été décrit par MoRGAN et al. (1979). La taille de l'échantillon donc la faible quantité de composés injectés n'impose pas l'emploi d'un diviseur.

Ce procédé offre, en outre, la possibilité d'introduire directement le matériel à étudier dans l'injecteur en effectuant un minimum de manipulations. Ce système peut donc s'appliquer aisément aux glandes mises en évidence chez des Insectes non sociaux (BIER et al., 1978, Descoins et al., 1978), ainsi qu'à certaines espèces pour lesquelles les phéromones ne sont pas synthétisées à partir de formations glandulaires (CHARARAS et al., 1980). En l'absence de solvant, les composés ne sont pas dilués et détectés quelle que soit leur polarité moléculaire, les risques de pertes par évaporation sont restreints; aussi les chromatogrammes obtenus apparaissent complexes. Inversement, les inévitables dissections influent fortement sur le niveau de la détection et peuvent nuire à la sensibilité de l'appareil. 

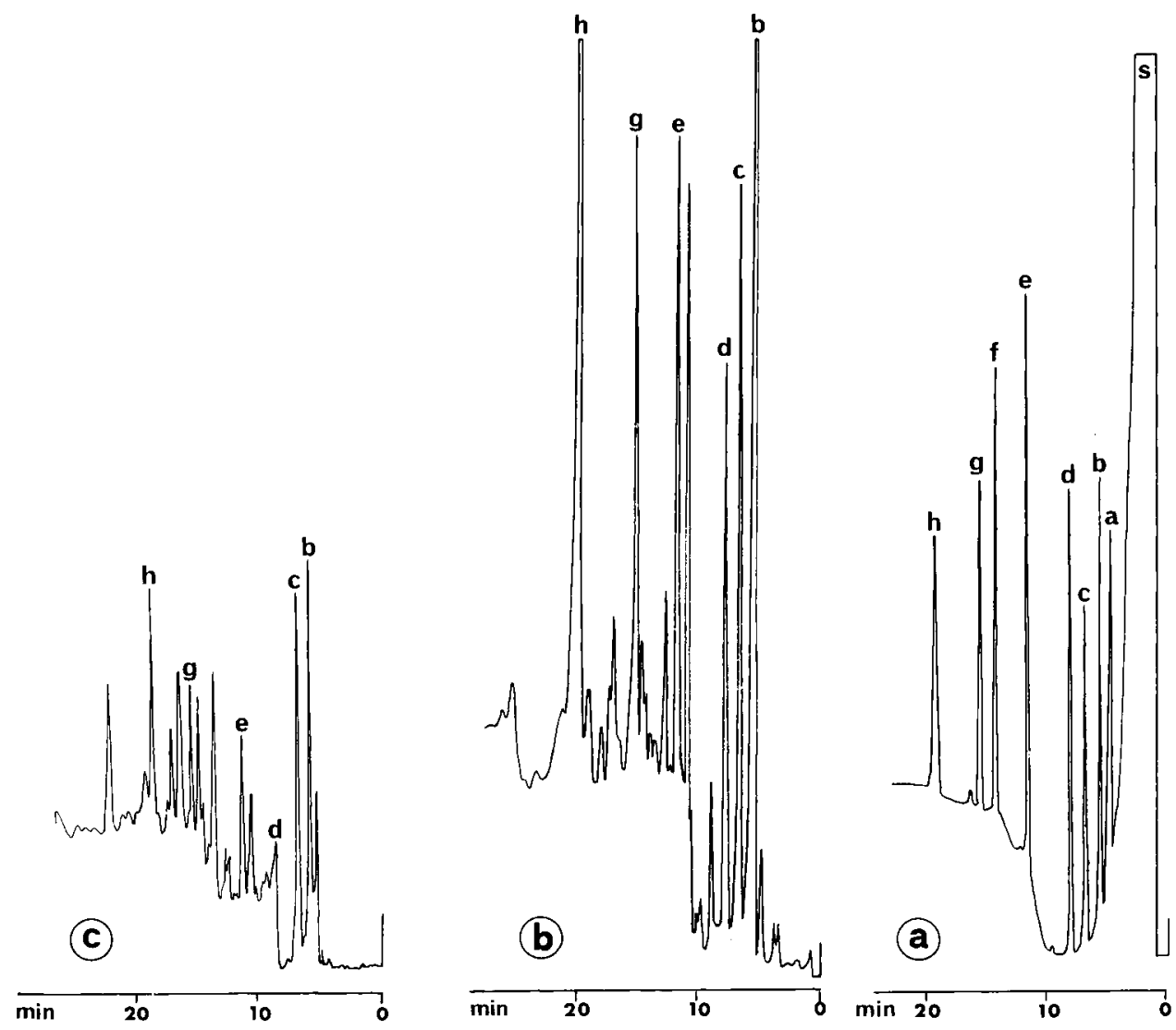

FIG. 5. - Chromatogrammes obtenus avec ce dispositif. a) solution standard.

$a$ : butyl acétate; $b:$ acétate d'isoamyle; $c:$ alcool isoamylique; $d:$ hexyl acétate;

$e:$ nonanol; $f$ : decyl-acétate; $g$ : benzyl acétate; $h:$ alcool benzylique; $s:$ solvant (hexane). b) 1 membrane poilue.

c) 20 glandes de Koschewnikow.

FiG. 5. - Chromatograms obtained with this device. a) standard solution.

$a:$ butyl acetate; $b$ : isoamyl acetate; $c:$ isoamylic alcohol; $d:$ hexyl acetate;

$e:$ nonanal; $f:$ decyl-acetate; $g:$ benzyl acetate; $h:$ benzylic alcohol; $s:$ solvent (hexane) b) 1 hairy membrane.

c) 20 Koschewnikow glands. 


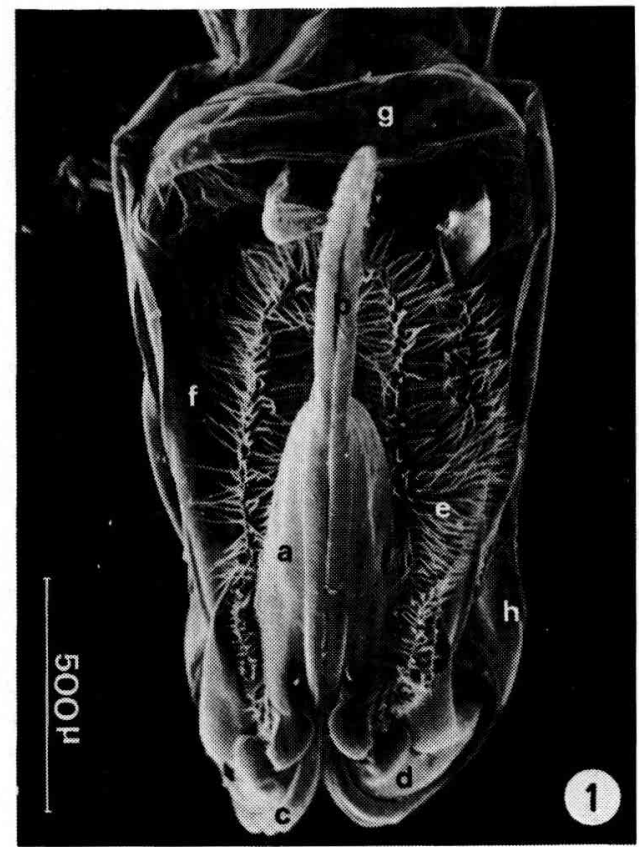

Photographie 1. - Détail du dard en vue ventrale. Pното 1. - Detailed ventral view of the sting.

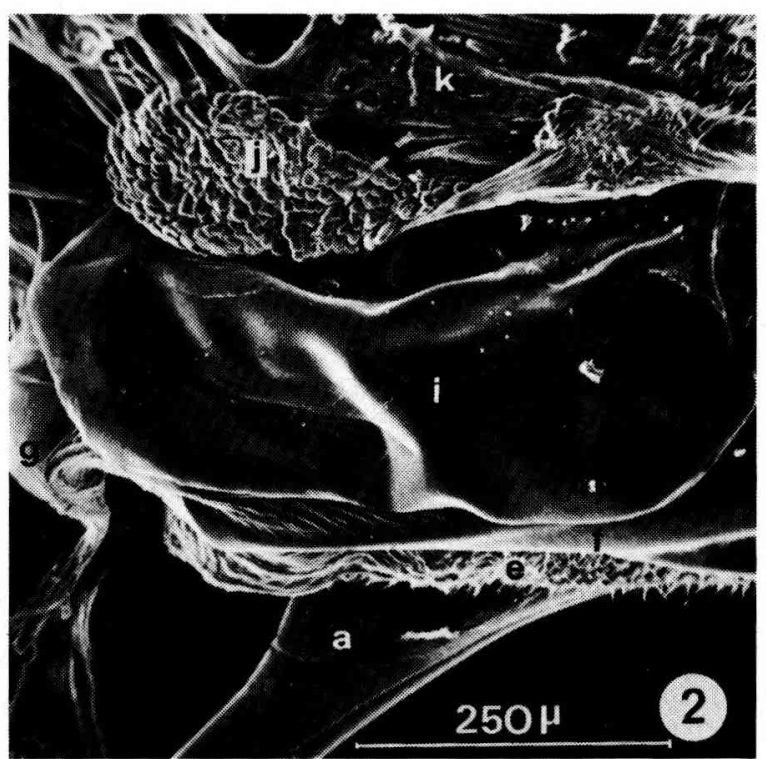

Photographie 2. - Détail du dard en vue latérale droite. Pното 2. - Detailed lateral right view of the sting. 
Les travaux effectués ici sur l'Abeille ouvrière ont pour but de déterminer l'origine et la nature des composés volatiles constituant la substance d'alarme en vue de tester leur activité biologique. Les chromatogrammes obtenus avec la membrane poilue et les glandes de Koschewnikow y révèlent la présence commune de nombreux produits dont l'acétate d'iso-amyl, composant actif et abondant de la phéromone d'alarme du dard d'ouvrière (Boch, Shearer et Stone, 1962).

Les importantes différences quantitatives observées entre membrane poilue et glandes de Koschewnikow sont attribuables aux pertes durant les dissections mais surtout à l'absence de véritable réservoir intraglandulaire (HEMSTEDT, 1969).

Ceci tend à confirmer l'hypothèse de GHENT et GARY sur l'origine de la substance d'alarme et précise le rôle des deux formations étudiées : élaboration de la phéromone au niveau des glandes, rétention et émission par la membrane poilue.

L'analyse montre en plus l'existence de composés non identifiés, dont certains, peu volatils, ne sont sans doute pas les plus actifs.

Reçu pour publication en juillet 1981 . Eingegangen im Juli 1981.

\section{ZUSAMMENFASSUNG \\ GASCHROMATOGRAPHIE DER FLÜCHTIGEN VERBINDUNGFN DER PHEROMONDRÚSEN DER BIENEN : METHODEN EINER DIREKTANALYSE}

Die Untersuchung der Verbindungen, die als Pheromone in das Leben der Insektenstaaten eingreifen, gestaitet sich wegen der Flüchtigkeit dieser Verbindungen schwierig. Um Verluste während der Manipulationen $\mathrm{zu}$ vermeiden, wurde ein Einlass-System entwickelt, das für alle Modelle von Chromatographen adaptiert werden kann. Es besteht aus einer Einführungskanüle, die durch das Septum in den Kopf der Säule führt, und die von dem Vektorgas durchströmt wird. Diese Kanüle ist mit einem Gefäss verbunden, in das verschlossene Ampullen mit den Proben eingeführt werden können. Mittels eines Kolbens können die Ampullen in die Einführungskanüle geschoben und im Kopf der Säule zerbrochen werden.

Diese Vorrichtung, deren Montage und Reinigung einfach ist, wurde bei der Analyse der Alarmsubstanzen des Stachels der Arbeitsbienen erprobt. Nach GHENT und GarY (1962) würde dieses Pheromon von den Koschewnikow-Drüsen gebildet und am Niveau der Haarmenbran abgegeben. In den angestellten Versuchen mit diesen beiden Organen wurde in ihnen gemeinsam das Vorhandensein zahlreicher flüchtiger Verbindungen nachgewiesen, darunter Isoamylacetat, die Hauptkomponente des Alarmpheromons. Sein Weg konnte wie folgt bestimmt werden :

- Bildung des Alarmpheromons in den Koschewnikow-Drüsen;

- Speicherung und Abgabe durch die Haarmembran.

\section{SUMMARY}

\section{GAS CHROMATOGRAPHY OF VOLATILE COMPOUNDS FROM PHEROMONAL GLANDS OF HONEYBEES : METHODS OF DIRECT ANALYSIS}

The study of pheromonal compounds in the lives of insect societies is made difficult by their volatile character. To avoid losses during manipulations, a direct introducing system adaptable to every 
chromatograph model has been devised. It includes an introducing needle adapted through the septum at the column head and traversed by the vector gas. This needle is connected to a cock and permits the putting in place of sealed ampoules containing the samples. A piston pushes the ampoules into the introducing needle and breaks them at the column head. This device, which is easy to mount and clean, has been tested in the analysis of the sting alarm substance of the honeybee worker. According to GHENT and GARY (1962), this pheromone emitted at the hairy membrane would be elaborated by the Koshevnikov glands. The tests carried out with two formations show the common presence of numerous volatile compounds including isoamyl acetate, the main compound of the alarm pheromone, and specify their role in this way:

- elaboration of the alarm pheromone by the Koshewnikov glands, and

- retention and emission by the hairy membrane.

\section{BIBLIOGRAPHIE}

BERGSTRÖM G., 1973. - Studies on natural odoriferous compounds. Use of a pre-column tube for the quantitative isolation of natural volatile compounds for gas chromatography/mass spectrometry. Chemica scripta, 4, 135-138.

Bierl B. A., Beroza M., Collier C. W., 1970. - Potent sex attractant of the gipsy moth, its isolation, identification and synthesis. Science, 170, 87-89.

Blum M. S., Fales H. M., Tucker K. W. and Collins A. M., 1978. - Chemistry of the sting apparatus of the worker honeybee. J. Apicultural Res., 17, 218-221.

Boch R., SHEARER D. and Stone B. C., 1962. - Identification of iso-amyl-acetate component in the sting pheromone of the honeybee. Nature, Lond., 195, 1018-1020.

Butenandt S. A., Beckmann R., Stamm O. and Hecker E., 1959. - Über den Sexuallockstoff des Seidenspinner Bombyx mori. Reindarstellung und Konstitution. Z. Naturforsch, 14, 283-284.

Chararas C., Riviére J., Ducauze C., Rutledge D., Delpui G. et Cazelles M.-T., 1980. Bioconversion d'un composé terpénique sous l'action d'une bactérie du tube digestif de Phloeosinus armatus (Coléoptère Scolytidae). C. R. Acad. Sc., D, 291; 2, 299-302.

Descoins C., Priesner E., Gallois M., Arn H. et Martin G., 1978. - Sur la sécrétion phéromonale des femelles vierges de Mamestra brassicae L. et Mamestra oleracea L. C. R. Acad. Sci., Paris (D), 286, $77-80$.

Descoins Ch. et M. Gallois, 1979. - Analyse directe par chromatographie en phase gazeuse des constituants volatiles présents dans les glandes à phéromones des femelles de Lépidoptères. Ann. Zool. Écol. Anim., 11, 521-532.

Ghent R. L. et Gary N. E., 1962. - A chemical alarm releaser in honeybee stings. Psyche, 69, 1-6.

Hemstedt H., 1969. - Zum Feinbau der Koschewnikowshen Drüse bei der Honigbiene (Apis mellifera L.). Zeitschrift für Morphologie der Tiere, 66, 51-72.

MAschwitz U., 1964. - Alarm substances and alarm processes for danger in social. Hymenoptera. $Z$. Vergl. Physiol., 47, 596-655.

Morgan E. D., Evershed R. P. and TyLeR R. C., 1979. - Gas chromatographic detection and structure analysis of volatile pheromones in Insects. J. Chrom., 11, 605-610.

Ställberg-Stenhagen, 1972. - Studies on natural odoriferous compounds. Splitter-free all glass intake system for glass capillary gas chromatography of volatile compounds from biological material. Chemice Scripta, 2, 97-100. 